\title{
(A)
}

\section{Directrices para el diseño y la construcción de videojuegos serios educativos}

\section{Guidelines for the Design and Construction of Serious Educationran} Video Games

Diretrizes para o design e construção de videogames educacionais sérios

\author{
Gabriel Elías Chanchí-Golondrino* (iD) orcid.org/0000-0002-0257-1988 \\ María Clara Gómez-Álvarez** (iD) orcid.org/0000-0002-4355-2978 \\ Luz Marina Sierra-Martínez ${ }^{* * *}$ orcid.org/0000-0003-3847-3324
}

\author{
Para citar este artículo \\ Chanchí-Golondrino, G. E., Gómez-Álvarez, M. C. y Sierra-Martínez, L. M. (2022). Directrices para el diseño y la \\ construcción de videojuegos serios educativos. Revista Colombiana de Educación, 1(84), 1-22. \\ https://doi.org/10.17227/rce.num84-12759
}

Fecha de recepción: 31/10/2020

Fecha de recepción: 25/01/2021

\section{(C) $(1)(9)$}

* Doctor en Ingeniería Telemática, Universidad del Cauca, Colombia. Profesor de la Facultad de Ingeniería - Universidad de Cartagena, Colombia. Correo: gchanchig@unicartagena.edu.co

** Doctora en Ingeniería, Sistemas e Informática, Universidad Nacional de Colombia. Profesora de la Facultad de Ingenierías - Universidad de Medellín, Colombia. Correo: mcgomez@udem.edu.co

*** Doctora en Ingeniería Telemática, Universidad Nacional de Colombia. Profesora del Departamento de Sistemas de la Universidad del Cauca, Colombia. Correo: Isierra@unicauca.edu.co 


\author{
Palabras clave: \\ aprendizaje; orientación; \\ video juegos; satisfacción; \\ motivación; aprendizaje \\ basado en juegos
}

\author{
Keywords: learning; \\ guidance; video games; \\ satisfaction; motivation; \\ game based learning
}

\section{Resumen}

En los últimos años una de las temáticas más difundidas en diferentes contextos de aplicación es el aprendizaje basado en juegos a través de los juegos serios que motivan a los usuarios y, por tanto, a medida que su uso se masifica es conveniente articular de mejor forma los juegos serios en el contexto educativo, de tal manera que, posibiliten el aprendizaje y el desarrollo de competencias definidas en los planes de estudio y se diseñen centrados en el usuario. En este artículo, se propone como contribución un conjunto de cincuenta y una orientaciones para el diseño, construcción y evaluación de videojuegos serios educativos, las cuales incluyen aspectos relacionados con usabilidad, aprendizaje y e juego. Las directrices fueron empleadas para inspeccionar el videojuego lightbot, con el fin de determinar los aspectos de diseño a mejorar en dicho videojuego. Las directrices propuestas en este trabajo pretenden vincularse en el proceso de desarrollo y en las metodologías para la construcción de videojuegos, de cara a propiciar la generación de videojuegos serios educativos centrados en el usuario, satisfactorios y más pertinentes al contexto educativo deseado.

\section{Abstract}

Game-based learning via serious games is among the most widespread themes over recent years within a range of application contexts. Learning by means of these games motivates users and, as their use burgeons, it makes good sense to clearly articulate serious games in the educational context so that they enable learning and development of skills defined in study plans, while taking care to ensure that such serious game design is user-centered. A set of fifty-one guidelines is herein proposed for the design, construction, and assessment of serious educational video games. The guidelines, which include aspects related to usability, learning, and play, were used to take a closer look at the LightBot coding-based puzzle game in order to determine design aspects that might be improved. The intention is that these guidelines might be incorporated in the development process and methodologies for the video game construction to foster the creation of serious user-centered educational video games that are rewarding and pertain more closely to the desired educational context.

\section{Resumo}

Nos últimos anos, um dos temas mais difundidos em diferentes contextos de aplicação é a aprendizagem baseada em jogos por meio de jogos sérios que motivam os usuários e, portanto, à medida que seu uso se torna mais difundido, é conveniente articular melhor os jogos sérios no contexto educacional, em de forma a permitir a aprendizagem e 0 desenvolvimento das competências definidas nos planos de estudo e desenhadas centradas no utilizador. Neste artigo, propõe-se como contribuição um conjunto de cinquenta e uma orientações para o projeto, construção e avaliação de videogames educacionais sérios, que incluem aspectos relacionados à usabilidade, aprendizagem e jogo. As diretrizes foram usadas para inspecionar o videogame lightbot, a fim de determinar os aspectos de design a serem melhorados no referido videogame. As diretrizes propostas neste trabalho pretendem estar vinculadas no processo de desenvolvimento e nas metodologias para a construção de videogames, a fim de promover a geração de videogames educacionais sérios voltados para o usuário, satisfatórios e mais relevantes para o contexto educacional desejado. 


\section{Introducción}

Una de las herramientas tecnológicas más populares en la actualidad son los videojuegos. Estos se entienden como plataformas creadas para el entretenimiento que permiten en gran medida satisfacer necesidades individuales relacionadas con el ocio; no obstante, ofrecen grandes potencialidades para el desarrollo de actividades de formativas (Calvo-Ferrer, 2018). Dentro del campo de los videojuegos encontramos los juegos serios (Zhu et al., 2020), que han sido ampliamente difundidos en diferentes contextos de aplicación, como salud, rehabilitación, educación, política, defensa, marketing, entre otros. Su aplicación se soporta en el hecho de que este tipo de videojuegos son una herramienta educativa y altamente motivacional con la capacidad de cambiar las actitudes de los usuarios, desarrollar habilidades y despertar conciencia acerca de estos campos específicos (Calvo-Morata et al., 2020; Moizer et al., 2019). Los juegos serios son más que una categoría específica de los video juegos (aventura, estrategia, deportes, etc.) y pueden definirse como juegos digitales creados con la intención de entretener y alcanzar al menos un objetivo adicional (Dörner et al., 2016). También, según Djaouti et al. (2011), los juegos serios son videojuegos cuyo propósito primario no es el entretenimiento y la diversión. Asimismo, según Da Silva Simões y Ferreira (2011), este tipo de videojuegos permiten el desarrollo de simulaciones que resultan más atractivas de jugar, al tiempo que ofrecen actividades que favorecen la asimilación de prácticas y habilidades psicomotoras.

Teniendo en cuenta lo anterior, en este tipo de videojuegos el interés principal va más allá del entretenimiento. Algunas de las características que los diferencian de los videojuegos comerciales son: están destinados para la educación, el entrenamiento de habilidades, la comprensión de habilidades complejas en diferentes contextos de aplicación y pueden ser usados en el ámbito de la publicidad; están vinculados o abordan una temática de la realidad; constituyen un ambiente en el que se permite la práctica segura de las habilidades de los aprendices y hay diferentes tipos de interés en sus contenidos dependiendo del contexto de aplicación en el que se desarrollen (política, marketing, economía, religión, entre otros) (Marcano, 2008). De acuerdo con lo presentado en Aguilar et al. (2018), los juegos serios pueden clasificarse en cuatro categorías: advergame, edutainment, edumarket y simulation game. Los advergames se emplean en la promoción de productos y servicios, de tal modo que involucran el producto dentro de la historia del juego; los edutainment tienen por objetivo el desarrollo de competencias educativas aprovechando el entretenimiento y la inmersión; los videojuegos tipo edumarket combinan aspectos relacionados con los advergame y los edutainment, de tal modo que muestran información sobre el funcionamiento de los mercados, generando atracción hacia ellos. Finalmente, los juegos de simulación buscan que el jugador adquiera o ejercite ciertas habilidades en el contexto de situaciones simuladas. Del mismo modo, según otros autores se pueden distinguir dos categorías principales que agrupan los diferentes tipos de juegos serios: advergame y edutainment (López Raventós, 2016).

Dadas las características propias de los videojuegos serios tipo edutainment, su aplicación en el contexto educativo tiene un gran potencial de cara al desarrollo de competencias 
educativas y resultados de aprendizaje en diferentes niveles de educación. En este sentido, los juegos serios tipo edutainment presentan diferentes contenidos educativos de la forma más lúdica posible, buscando hacer divertido aquello que no parece serlo (López Raventós, 2016) y facilitando la apropiación de algunos conceptos complejos por parte de los estudiantes en el aula de clase. Del mismo modo, los videojuegos serios aplicados al contexto educativo universitario buscan servir de apoyo a los docentes en cuanto a la formación de futuros profesionales en los retos de desempeño exigidos por un mundo globalizado, complejo y de cambio técnico acelerado (Rojo y Dudu, 2017). A pesar de las potencialidades de los videojuegos serios en el contexto educativo, aún existen desafíos en cuanto a su implementación en escenarios educativos, dada la estrecha relación que debe existir con el proceso educativo (Darwesh, 2015). En este mismo sentido, la adopción de juegos serios en una amplia gama de contextos educativos y de capacitación genera una necesidad creciente para comprender y evaluar su efectividad desde la perspectiva de la experiencia de usuario, con el fin de mejorar la interacción entre el usuario y el videojuego (Moizer et al., 2019). De este modo, conviene que los videojuegos serios tengan en cuenta pautas y directrices tanto a nivel educativo como a nivel de calidad de uso, usabilidad y jugabilidad, con el fin de permitir que los estudiantes centren su atención en los retos y objetivos de aprendizaje propuestos teniendo en cuenta los procesos de aprendizaje, no en las dificultades en el manejo de los videojuegos (comandos complicados, interfaz poco intuitiva, tiempo de respuesta inadecuado, excesiva o insuficiente cantidad de información, fallas en la navegación, etc.) (Savazzi et al., 2018; Tolentino et al., 2011).

La usabilidad puede ser entendida como la calidad de la interacción entre el usuario y el sistema y está compuesta por atributos tales como facilidad de aprendizaje, eficiencia, facilidad de recordación, manejo de errores y capacidad de producir satisfacción en el usuario final (Chanchí et al., 2020; Tolentino et al., 2011). Del mismo modo, la usabilidad se puede definir como la eficacia, eficiencia y satisfacción con la que un usuario puede alcanzar objetivos específicos en un contexto de uso específico (Finstad, 2010; González-Sánchez et al., 2012). Dentro de las principales ventajas de la usabilidad se encuentra la posibilidad de que las empresas del sector software sean más competitivas y los usuarios finales de los desarrollos sean más productivos (Madhavan y Alagarsamy, 2013; Mascheroni et al., 2012; Sánchez, 2011). Por otra parte, la experiencia de usuario va más allá de la usabilidad y está comprendida en el servicio en el cual se enmarca el software, es decir, se enfoca en la interacción de los clientes con los productos, sistemas y servicios. De este modo, mientras la usabilidad se enfoca en la satisfacción en el uso del software, la experiencia de usuario involucra la satisfacción en el servicio (González-Sánchez et al., 2012; Jang y Yi, 2019; Kramer, 2012).

Con el fin de mejorar la calidad de interacción, así como propiciar el desarrollo de competencias educativas dentro de los videojuegos serios tipo educativo o edutainment, se hace necesario recopilar, adaptar, sintetizar y clasificar diferentes pautas y directrices de diseño a partir de la literatura, las cuales tengan en cuenta aspectos como la calidad de uso, la experiencia de usuario, el proceso de enseñanza-aprendizaje y la jugabilidad. Las directrices propuestas pretenden servir de apoyo mediante su articulación en el proceso de desarrollo de videojuegos serios educativos centrados en el usuario, de manera específica en las fases de diseño y evaluación, de tal modo que se contribuya con la calidad de la interacción y la apropiación de los conceptos educativos abordados por los videojuegos serios. 
A continuación, se presenta un conjunto de trabajos que se tuvieron en cuenta como referencia para la definición de las directrices para el diseño de videojuegos serios tipo edutainment.

En Pinelle et al. (2008), se propone un conjunto de diez heurísticas de usabilidad para videojuegos, las cuales fueron obtenidas del análisis de 108 revisiones realizadas por usuarios en el portal GameSpot. A partir de las revisiones analizadas se obtuvo un conjunto de problemas comunes, que fueron organizados y clasificados dependiendo del tipo de problema. Las heurísticas planteadas por los autores sugirieron respuestas a los problemas identificados. Estas fueron: respuesta consistente a las acciones del usuario; personalización de configuraciones multimedia (audio y video) y dificultad del juego; comportamiento razonable para las unidades controladas por computador; vistas sin obstrucción apropiadas para las acciones del usuario; permitir saltar contenido frecuente, repetitivo y no jugable; proveer entradas intuitivas y personalizables; proveer controles fáciles de manejar y con un adecuado nivel de sensibilidad; información sobre el estado del juego; proveer instrucciones, entrenamiento y ayuda; representaciones visuales fáciles de interpretar. Si bien estas heurísticas han sido propuestas inicialmente para el contexto de los videojuegos, muchos aspectos de los aquí presentados pueden extrapolarse al escenario de los videojuegos serios.

En Paliokas et al. (2011), se propone un videojuego educativo denominado PlayLogo 3D, que fue diseñado para el desarrollo de habilidades básicas de programación en niños de seis a trece años. Además, para la evaluación del videojuego desde la perspectiva de la usabilidad se propuso un instrumento que toma en consideración tres categorías de heurísticas de usabilidad (usabilidad del juego, aspectos del juego, aspectos educativos y pedagógicos), a cada una de las cuales se les definieron unos criterios expresados en términos de preguntas. Los criterios definidos pueden servir de referencia para el diseño de videojuegos educativos y su extrapolación al contexto de los videojuegos serios tipo edutainment.

En Annetta et al. (2011) se propone una rúbrica de evaluación para videojuegos serios educativos, en la cual se definen quince aspectos por considerar tanto en las fases de diseño como de evaluación, los cuales incluyen elementos pedagógicos y de experiencia de usuario. La rúbrica definida por los autores está compuesta por las siguientes categorías: prólogo, nivel de práctica, interactividad, retroalimentación, identidad, inmersión, frustración Deseable, Manipulación, Incremento de la complejidad, Reglas, Aprendizaje informado, Efectividad pedagógica, Eficiencia en la lectura y comunicación. Los aspectos definidos incluyen tanto elementos pedagógicos como de usabilidad, que pueden ser agrupados y sintetizados en un conjunto reducido de categorías, dada la relación existente entre estos.

En Darwesh (2015), se menciona que, a pesar de la potencialidad en los videojuegos serios educativos, estos no han sido ampliamente utilizados en el contexto educativo debido a la falta de articulación de los videojuegos serios con el proceso educativo. A partir de lo anterior, se proponen seis conceptos fundamentales por considerar en el diseño y construcción de juegos serios con propósito educativo. Dentro de los conceptos propuestos están: escenario educativo, interactividad, seguimiento del usuario, entusiasmo, puntuación y aprendizaje. A partir de los anteriores conceptos, el autor plantea la posibilidad de propiciar el diseño de videojuegos serios educativos que propendan por el desarrollo del concepto de educación adaptativa. 
En Fitchat y Jordaan (2016), se propone un conjunto de diez heurísticas definidas para evaluar la experiencia de usuario en videojuegos serios. Las heurísticas planteadas en este trabajo fueron obtenidas a partir de las entrevistas realizadas a un grupo de usuarios que desarrollaron un test de usuario con el videojuego serio educativo StoryTimes, que define un conjunto de actividades lúdicas para la apropiación del concepto de tablas de multiplicación. Las heurísticas definidas fueron expresadas en términos de lo que los usuarios desearían que el juego tuviera, así: los jugadores quieren conveniencia; los jugadores quieren estar encantados e inmersos en el juego; los jugadores desean aprender sin esfuerzo; los jugadores quieren ser motivados constantemente por el juego; los jugadores quieren practicar; los jugadores quieren ser recompensados por sus logros; los jugadores desean utilizar todos sus sentidos; los jugadores quieren conocer s u progreso; los jugadores necesitan ser guiados a lo largo del juego; los jugadores requieren personalización en el juego.

En Savazzi et al. (2018), se propone un modelo utilizado para evaluar la usabilidad de videojuegos serios en el área de neuro-rehabilitación. La definición del modelo de evaluación de usabilidad es una adaptación de las heurísticas de Nielsen en un conjunto de ocho principios para el diseño y la construcción de videojuegos serios: consistencia, visibilidad, relación entre el sistema y el mundo real, memoria, retroalimentación, flexibilidad y control, manejo de errores y lenguaje. El artículo presenta los resultados de un estudio de caso en el que se usan las heurísticas definidas para evaluar un videojuego del área de la neuro-rehabilitación, y se obtuvo un conjunto de aspectos por mejorar denle cuanto al diseño.

Abdellatif et al. (2018) mencionan la necesidad de definir un conjunto de heurísticas que determinen el diseño y la evaluación de videojuegos serios, con el fin de mejorar la experiencia de uso. A partir de lo anterior, en este trabajo se propone un marco de cinco dimensiones para la evaluación de videojuegos serios, en donde cada una está conformada por un conjunto de criterios que permiten verificar su cumplimiento. Dichas dimensiones son: usabilidad, motivación, compromiso, experiencia de usuario y comprensibilidad. A partir de las dimensiones y criterios definidos, se desarrolló una evaluación del videojuego de programación Robocode.

En Moizer et al. (2019) se propone la adaptación de un modelo de evaluación de la experiencia de usuario en videojuegos serios de carácter holístico, la cual está conformada por dimensiones, atributos y criterios (definidos en términos de preguntas). Las dimensiones incluidas en el modelo propuesto son: experiencia en el juego, experiencia de aprendizaje, adaptabilidad, usabilidad y fidelidad. El modelo se empleó para la evaluación del juego serio S-Cube, que se basa en roles y tiene por objetivo el desarrollo de habilidades blandas en trabajadores de empresas de carácter social. Los autores concluyen que el modelo puede servir de referencia para mejorar la experiencia de usuario en videojuegos serios de diferentes contextos de aplicación.

En Vee Senap e Ibrahim (2019) se presenta una revisión de literatura centrada en la identificación de heurísticas para la evaluación de videojuegos educativos móviles, que puedan ser empleadas en la conducción de evaluaciones heurísticas sobre videojuegos, con el fin de mejorar aspectos referentes a la interacción. De este modo, a nivel de la usabilidad en el contexto de los videojuegos se identificó la presencia de los siguientes componentes en diferentes trabajos: desafíos, fantasía, curiosidad, interfaz, mecánicas, jugabilidad, historia, usabilidad, movilidad, entretenimiento y social. Igualmente, con respecto a los componentes 
de usabilidad en videojuegos educativos se identificaron a partir de diferentes trabajos los siguientes componentes: interfaz, pedagógico, contenido, jugabilidad, multimedia, entretenimiento, usabilidad, mecánicas, personajes y movilidad. Asimismo, en cuanto a los componentes claves de usabilidad en aplicaciones móviles se identificaron: usabilidad, interfaz y control. Los componentes identificados por los autores permiten la definición de heurísticas, pautas y directrices que pueden ser extrapoladas al contexto de los videojuegos educativos.

En Maheu-Cadotte et al. (2020) los autores realizan un comparativo entre las características de los simuladores virtuales y los videojuegos serios en el contexto de la salud. A partir de la identificación y el estudio de los elementos diferenciadores en estos dos tipos de aplicaciones, los autores definieron un conjunto de heurísticas para guiar el proceso de diseño y construcción de simuladores virtuales y videojuegos serios en el contexto de la salud. En cuanto a los juegos serios educativos aplicados al contexto de la salud, los autores proponen las siguientes heurísticas: autenticidad, diversión, fantasía, retroalimentación, estructura e inquietudes. Dentro de la categoría Diversión se destacan las subcategorías Logros, Competencia, Control y Curiosidad. A modo de conclusión, los autores resaltan que las categorías de Diversión y Fantasía están presentes en los videojuegos serios, mas no en los simuladores virtuales.

A partir de los trabajos presentados en esta sección, se concluye que existen diferentes propuestas de heurísticas y pautas para el diseño y la construcción de usabilidad, las cuales tienen en cuenta aspectos como la usabilidad, la experiencia de uso y los elementos pedagógicos y de aprendizaje. Estas heurísticas y pautas pueden ser clasificadas y agrupadas en un conjunto de directrices puntuales organizadas por categorías que permitan apoyar el proceso de desarrollo de videojuegos serios tipo edutainment.

El resto del artículo está organizado de la siguiente forma: la sección 2 presenta la metodología considerada para el desarrollo del presente trabajo; en la sección 3 se muestran los resultados del trabajo, específicamente las directrices adaptadas y clasificadas para el diseño y la construcción de videojuegos serios de tipo educativo, así como un estudio de caso en el cual se realiza una inspección sobre un videojuego serio tipo edutainment con el fin de verificar el cumplimiento de las directrices propuestas en este; finalmente, la sección 4 presenta las conclusiones y los trabajos futuros derivados de esta investigación.

\section{Métodología}

En el desarrollo de la presente investigación, se definieron cuatro fases, a saber: F1) conceptualización de los juegos serios en el contexto educativo, F2) exploración de heurísticas para juegos serios, F3) adaptación y clasificación de las directrices para juegos serios en el contexto educativo, y finalmente, F4) estudio de caso (véase la figura 1). 


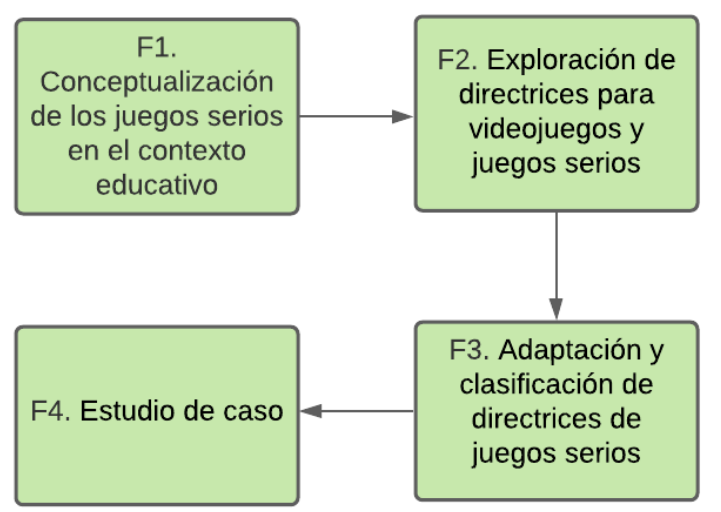

Figura 1. Metodología considerada para el desarrollo del estudio. Esta metodología permitió proponer directrices específicas para el diseño, la construcción y la evaluación de videojuegos serios en contextos educativos

Fuente: elaboración propia.

En la fase $F 1$ se realiza la conceptualización de los juegos serios aplicados al contexto educativo, con el fin de identificar sus características principales. En la fase F2, se exploraron a partir de la literatura diferentes directrices para el diseño de videojuegos y juegos serios, las cuales fueron adaptadas y clasificadas para el contexto de los juegos serios en el ámbito educativo en la fase F3. Finalmente, a partir de las directrices definidas, en la fase F4, se condujo una inspección sobre un videojuego serio clasificado como de tipo educativo (Lightbot).

\section{Resultados}

En esta sección, se presenta un conjunto de directrices para el diseño y la construcción de videojuegos serios para el contexto educativo, las cuales fueron obtenidas y adaptadas a partir de los trabajos presentados en la revisión del estado del arte, que involucran pautas, heurísticas, conceptos y cuestionarios de evaluación para videojuegos, juegos serios y videojuegos en el contexto educativo. Las directrices presentadas abordan temáticas como la usabilidad, el proceso de aprendizaje y los aspectos referentes al juego, por tanto fueron agrupadas en estas tres categorías, de acuerdo con lo propuesto por Paliokas et al. (2011). En cuanto a la usabilidad, se tuvieron en cuenta heurísticas relacionadas con la interacción en el ámbito de los videojuegos, las cuales generalmente son adaptadas a partir de las heurísticas propuestas por Nielsen. En lo referente a la categoría Aprendizaje, se tuvieron en cuenta aspectos relacionados con la incorporación del aprendizaje dentro de los videojuegos. Finalmente, en cuanto al aspecto del juego, se tomaron en consideración elementos como inmersión, motivación, seguimiento al jugador, retroalimentación, retos, logros, puntaje, retroalimentación, entre otros. Las directrices referentes a la usabilidad se identifican con el prefijo DU, las directrices relacionadas con el aprendizaje tienen el prefijo DA, y las directrices que comprenden los aspectos del juego tienen el prefijo DJ. En total se obtuvieron cincuenta 
y una directrices, organizadas de la siguiente manera: 15 de usabilidad, 13 de aprendizaje y 23 de juego (tabla 1).

Tabla 1. Directrices propuestas para videojuegos serios

\section{Categorías}

Usabilidad

\section{Directrices}

Du1. La interfaz del videojuego serio es intuitiva y fácil de utilizar para el jugador. Se ha hecho uso de metáforas visuales que resultan familiares al jugador.

DU2. El videojuego serio debe incluir un diseño consistente en las diferentes vistas en cuanto al uso de los colores, fuentes, disposición de los elementos en pantalla, lo cual propicia la inmersión del jugador en el juego.

DU3. El videojuego serio incluye una navegación y operación consistente, lógica y minimalista.

DU4. El videojuego serio incorpora en el escenario y las representaciones visuales elementos que se corresponden con el mundo real.

DU5. En la medida de lo posible, el videojuego serio debe evitar que el usuario memorice una gran cantidad de información referente a la historia y al modo de usar el juego.

DU6. El videojuego serio debe proveer información sobre el estado del juego en sus diferentes vistas.

DU7. Los diferentes elementos del videojuego serio, tales como personajes, objetos y elementos del escenario, deben tener un comportamiento predecible.

DU8. El videojuego serio debe usar comandos convencionales y personalizables con un nivel de respuesta y sensibilidad adecuado. 


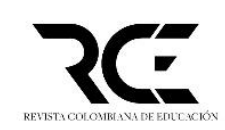

Categorías
Directrices para el diseño y la construcción de videojuegos serios educativos |

Gabriel Elías Chanchí-Golondrino María Clara Gómez-Álvarez Luz Marina Sierra-Martínez

DU9. El videojuego serio debe permitir al usuario tener control sobre el funcionamiento del juego, así como deshacer acciones indeseadas.

Du10. El videojuego serio se adapta a diferentes estilos y permite la personalización de las configuraciones de juego.

DU11. El videojuego serio debe posibilitar la configuración de opciones de audio y video.

DU12. El videojuego serio debe prevenir los posibles errores que un jugador puede cometer, y en caso de que el jugador cometa un error emplear mensajes claros que permitan al usuario volver a un estado estable.

DU14. El videojuego serio provee opciones de ayuda claras que permiten guiar al usuario sobre los comandos, modos de juego y posibles configuraciones.

DU15. El videojuego serio provee una ayuda contextual en los diferentes retos planteados, la cual orienta al usuario sobre el propósito de estos.

\footnotetext{
Aprendizaje

DA1. Para que un videojuego serio pueda resultar efectivo y con una buena divulgación en el contexto educativo debe partir de las competencias académicas formales de un curso determinado o basarse en ellas.

DA2. Los retos y actividades del videojuego serio están bien estructurados y organizados de acuerdo con las competencias del curso en el cual se enmarca el juego.

DA3. El escenario del videojuego serio es adecuado, consistente y relevante para el desarrollo de las competencias abordadas por los objetivos de aprendizaje.

DA4. El videojuego tiene definidos claramente para cada nivel y/o reto un objetivo de aprendizaje y un objetivo de juego, los cuales guardan consistencia entre sí.
} 


\section{Directrices}

DA5. El videojuego serio debe propender por la apropiación de las diferentes temáticas educativas a través del diseño de retos y niveles que le resulten divertidos al jugador.

DA6. El videojuego serio posibilita a través de sus retos el aprendizaje implícito del jugador, de tal modo que este olvide que está aprendiendo.

DA7. Los diferentes niveles y retos de los juegos serios permiten abordar implícitamente los contenidos educativos del aula de una forma simplificada y entretenida.

DA8. El videojuego serio está diseñado y estructurado para promover el aprendizaje autónomo por parte de los jugadores. Está diseñado bajo el enfoque de aprender haciendo.

DA9. El videojuego serio debe propiciar la sensación en el usuario de que el cumplimiento de los retos contribuye a su proceso de aprendizaje.

DA10. Los diferentes niveles o retos del videojuego serio son incrementales, de tal modo que las temáticas educativas abordadas en cada nivel contribuyen al cumplimiento de retos y niveles futuros.

DA11. El videojuego serio permite la recopilación de los datos referentes al desempeño del jugador en los diferentes retos (puntajes obtenidos) y opcionalmente permite la obtención de métricas útiles con las que el jugador pueda hacer un seguimiento a la relación entre objetivos de aprendizaje y objetivos del juego.

DA12. El videojuego serio permite el seguimiento del progreso del jugador en los diferente retos y niveles, de tal forma que este pueda visualizar y analizar su progreso en el cumplimiento de los objetivos de aprendizaje. 
DA13. Dependiendo del campo de aplicación del videojuego serio, el juego posibilita el trabajo colaborativo para la consecución de objetivos de aprendizaje colectivos. en los diferentes retos y niveles de este, del tal modo que el jugador tenga la sensación de perder la noción de tiempo y lugar.

DJ2. El videojuego serio mantiene la motivación del jugador cuando existen dificultades para superar los retos propuestos. De este modo, el videojuego busca reducir al máximo la retroalimentación negativa.

DJ3. El videojuego serio debe propiciar el uso y la estimulación de los diferentes sentidos a través de los contenidos multimedia usados en los diferentes niveles y retos.

DJ4. La retroalimentación e inmersión provistas por el videojuego serio en los diferentes niveles permiten crear en los jugadores la sensación de querer cumplir cada reto.

DJ5. El videojuego serio debe posibilitar que el jugador incremente su motivación y autoestima con el cumplimiento de los retos propuestos en los diferentes niveles.

DJ6. Los retos y actividades del videojuego serio, en la medida de lo posible, no deben ser repetitivos ni aburridos.

DJ7. El videojuego serio a partir de sus retos debe incluir progresivamente elementos novedosos que promuevan la curiosidad por seguir jugando.

DJ8. El videojuego debe posibilitar la visualización del progreso de los diferentes jugadores con el fin de propiciar la motivación del jugador.

DJ9. El videojuego serio debe dar la posibilidad de superar los retos de cada nivel con diferentes grados de cumplimiento o 


\section{Directrices}

niveles de puntaje con el fin de que el jugador tenga una mayor motivación de superarse.

DJ10. Se sugiere el uso del tiempo como mecanismo para propiciar la diversión en los diferentes retos del juego.

DJ11. El videojuego serio debe proveer una introducción al juego y a los diferentes retos, que permita informar al jugador sobre los objetivos por cumplir.

DJ12. La narrativa del videojuego serio debe permitir conectar con los diferentes retos del juego.

DJ13. Se recomienda la incorporación de logros o recompensas para los diferentes retos pertenecientes a los niveles del videojuego serio.

DJ14. El videojuego brinda la posibilidad de entrenar las habilidades del jugador a través de un nivel de entrenamiento o dando la posibilidad de volver a jugar los retos.

DJ15. El videojuego posibilita la interacción con los objetos del entorno virtual y con otros personajes, de tal modo que las diferentes interacciones proporcionan como respuesta información para que el usuario cumpla los objetivos del juego.

DJ16. Las diferentes acciones realizadas en el videojuego serio tienen una retroalimentación, la cual está de acuerdo con las narrativas del juego y ayudan a orientar al usuario en el cumplimiento de los objetivos.

DJ17. El videojuego serio le permite al jugador escoger o personalizar un avatar que lo represente a lo largo de los diferentes retos y niveles.

DJ18. La historia del videojuego serio permite al usuario conectarse con los retos y temáticas abordadas en el juego. 


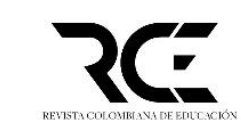

Categorías
Directrices para el diseño y la construcción de videojuegos serios educativos |

Gabriel Elías Chanchí-Golondrino

María Clara Gómez-Álvarez

Luz Marina Sierra-Martínez

DJ19. El videojuego debe hacer uso correcto del audio y la música de fondo, de tal forma que propicien la motivación y concentración del jugador en los diferentes niveles y retos.

DJ20. El videojuego serio debe ser flexible y permitir adaptarse a diferentes estilos de juego y de jugador.

DJ21. El videojuego serio promueve la competencia entre los jugadores a través de la presentación de los resultados de desempeño y el manejo de clasificaciones.

DJ22. Los textos empleados en las instrucciones y en la retroalimentación del videojuego serio son claros y adecuados para los usuarios objetivo del mismo. Además, estos textos pueden ser complementados mediante audios diseñados de manera pedagógica.

DJ23. Los diferentes mensajes presentados en el juego deben emplear un lenguaje claro y cercano al usuario.

Fuente: elaboración propia.

Una vez se presentaron las cincuenta y una directrices para el diseño y la construcción de videojuegos serios educativos (véase la tabla 1), se desarrolló un estudio de caso consistente en una inspección cualitativa de usabilidad. Se trata de un método mediante el cual un conjunto de evaluadores inspecciona y detecta los posibles problemas de diseño de un software de acuerdo a un conjunto de heurísticas, pautas o directrices (Enriquez y Casas, 2013). Los resultados de la inspección permiten determinar aspectos del software que se pueden mejorar con el fin de aumentar su calidad.

Como estudio de caso se realizó la inspección de usabilidad sobre el videojuego serio educativo Ligthbot (https://lightbot.com/flash.html), que tiene como objetivo el desarrollo de habilidades de lógica de programación (véase la figura 2). El videojuego cuenta con tres niveles: basics, procedures y loops, cada uno de los cuales tiene asociado un conjunto de retos propuestos, que involucran la creación de un algoritmo que permite programar los movimientos del robot para llegar de un origen a un destino. 


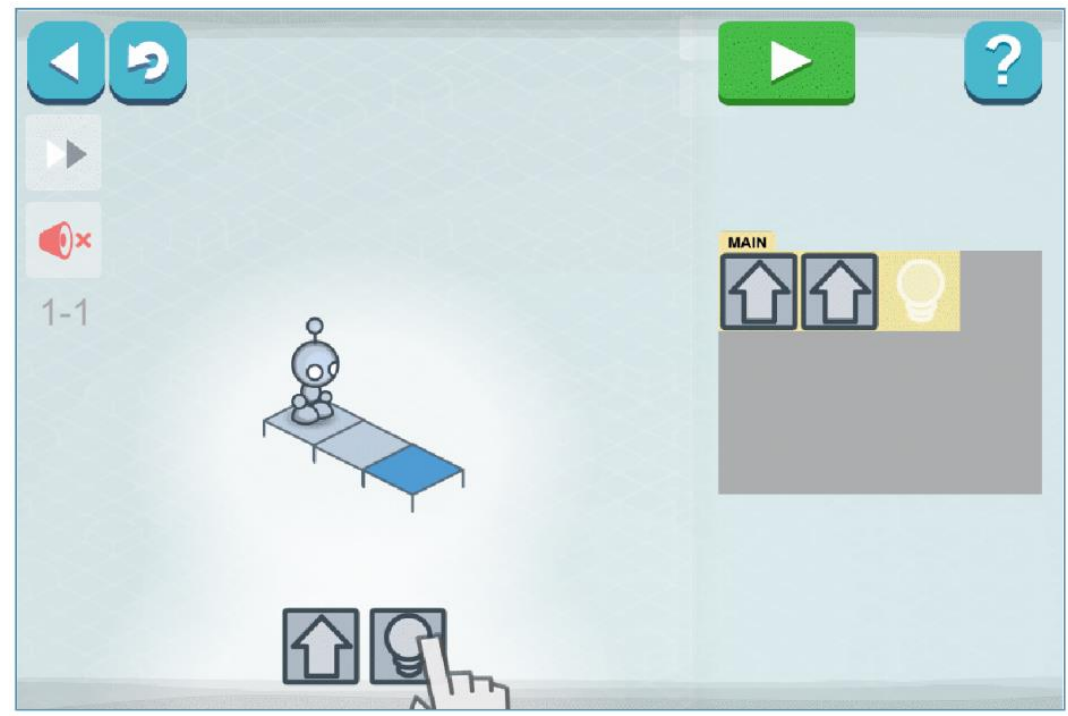

Figura 2. Videojuego Lightbot.

Fuente: tomado de https://lightbot.com/flash.html.

A partir de la inspección realizada al videojuego serio Ligthbot, se obtuvieron las siguientes recomendaciones para las tres categorías consideradas en las directrices propuestas: usabilidad, aprendizaje y juego (véase la tabla 2). Estas recomendaciones buscan mejorar la interacción, la jugabilidad y el refuerzo de aspectos de aprendizaje en el videojuego serio evaluado.

Tabla 2. Resultado de la prueba de inspección de usabilidad según las directrices propuestas

Categorías

Usabilidad

\begin{tabular}{l} 
Problemas \\
\hline Con el fin de mejorar la consistencia en las diferentes vistas del \\
juego, se recomienda trabajar con una sola gama de colores, ya \\
que existen diferentes colores (gris, azul, verde) en los botones \\
del videojuego serio (DU2). \\
Aunque el videojuego serio permite personalizar diferentes \\
idiomas del juego, al escoger el idioma español no se mantiene \\
la consistencia en los diferentes textos, dado que algunos \\
permanecen en inglés (por ejemplo, Main). En este sentido, se \\
recomienda mantener la consistencia en los textos traducidos \\
(Du2). \\
La orientación de las flechas que permiten programar el \\
movimiento del robot no es la misma con respecto a la dirección \\
\hline
\end{tabular}




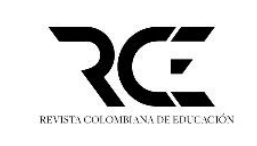

Categorías
Directrices para el diseño y la construcción de videojuegos serios educativos |

Gabriel Elías Chanchí-Golondrino María Clara Gómez-Álvarez Luz Marina Sierra-Martínez

\section{Problemas}

en la que este se mueve. Se recomienda seguir las convenciones del mundo real (DU4).

Es conveniente emplear íconos más claros y que se correspondan con las convenciones del mundo real para programar los movimientos de giro a la derecha y a la izquierda (DU4).

Se recomienda incluir en la barra de estado del juego aspectos tales como: el número de intentos, el tiempo empleado en cumplir cada reto y el identificador del reto en el que se encuentra el usuario (DU6).

Se recomienda la inclusión de comandos convencionales de teclado para la programación de la ruta y el movimiento del robot, dado que el juego solo puede operarse mediante el mouse (DU8).

Es aconsejable presentar la información de la ayuda en una forma unificada, debido a que al presionar la opción respectiva se muestran un conjunto de mensajes secuenciales, que no se pueden evitar hasta que se visualicen por completo. Esto hace que el jugador pierda el control sobre la interfaz del juego (DU9).

Se sugiere incluir un mensaje informativo cuando el jugador falle en la programación del camino del robot, de tal modo que exista una retroalimentación clara en la interacción entre el jugador y el juego (DU12).

Aunque el videojuego serio cuenta con ayuda al inicio de cada uno de los retos, en los cuales los mensajes de ayuda son presentados en ráfaga, se sugiere incluir una ayuda contextual para retos en los que el jugador se pueda estancar (DU15).

Aprendizaje Dado que el videojuego serio incluye tres temáticas: básico, ciclos y procedimientos, se recomienda incorporar en los niveles del juego temáticas intermedias que corresponden con el 


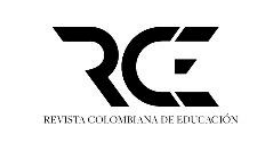

Categorías
Directrices para el diseño y la construcción de videojuegos serios educativos |

Gabriel Elías Chanchí-Golondrino María Clara Gómez-Álvarez Luz Marina Sierra-Martínez

contenido programático de los cursos de lógica de programación, como son instrucciones de decisión (DA1 y DA2).

Se sugiere incluir en el videojuego serio mensajes de retroalimentación ante aciertos o fallas en la programación de la ruta del robot, los cuales permitan al usuario percibir que el cumplimiento de los retos contribuye al proceso de aprendizaje (DA9).

Dado que el videojuego serio solo permite mostrar al jugador los niveles que este ha superado, pero no maneja puntajes o recompensas, se recomienda incluir puntajes, tiempos asociados a cada reto y niveles de cumplimiento de los retos, con el fin de poder realizar un seguimiento más adecuado del cumplimiento de los objetivos de aprendizaje (DA11 y DA12).

Se sugiere que el videojuego serio permita visualizar al jugador el nivel de cumplimiento de cada uno de los retos, con el fin de que pueda mejorar el desempeño en cada uno de estos (DA12). El juego solo muestra si se ha superado o no el reto.

Para mantener la motivación del jugador a la hora de superar un reto, se recomienda la inclusión de mensajes que lo motiven y lo retroalimenten mediante indicaciones sobre las posibles causas de que no haya cumplido el objetivo de cada reto (DJ2, DJ4 y DJ16).

Es necesario incluir mensajes cuando el jugador no cumpla un reto, al igual que otros que propicien la motivación y autoestima cuando haya superado un reto (DJ5, DJ16).

Se recomienda mejorar la presentación de estadísticas con respecto al grado de cumplimiento de cada uno de los retos del juego, dado que hasta el momento solo se muestra si el reto fue o no aprobado. Lo anterior implica la inclusión de puntajes, tiempos y métricas asociadas a estas variables (DJ8 y DJ9). 


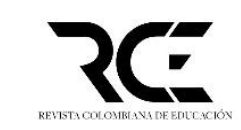

Categorías
Directrices para el diseño y la construcción de videojuegos serios educativos |

Gabriel Elías Chanchí-Golondrino María Clara Gómez-Álvarez Luz Marina Sierra-Martínez

\section{Problemas}

Se sugiere incluir el tiempo como mecanismo para evaluar el progreso y promover la diversión en cada reto del juego, dado que hasta el momento los retos son independientes del tiempo (DJ10).

Es aconsejable incluir en cada nivel, u opcionalmente en cada reto, una introducción que describa de manera clara el objetivo de aprendizaje o de juego que debe cumplir cada jugador (DJ11).

Se recomienda la incorporación de logros o recompensas para los diferentes retos aprobados por el jugador en cada uno de los niveles del juego (DJ13).

Se sugiere la inclusión de un nivel de entrenamiento, en el cual se describan aspectos como los íconos comunes con los que el jugador interactuará para que comprenda las mecánicas del juego (DJ14).

Aunque la narrativa del juego no está explícita, puede intuirse una vez se van superando los retos del juego. Sin embargo, con el fin de contextualizar y promover la inmersión del jugador, se sugiere la inclusión de la historia del juego (DJ18).

Para promover la competencia entre diferentes jugadores en el aula de clase, se recomienda la inclusión de los resultados y clasificaciones de desempeño. Como se mencionó, es necesario que el juego incluya los niveles de cumplimiento de cada reto, a partir de métricas como el tiempo, número de fallas, etc. (DJ21). Se recomienda complementar los mensajes de ayuda y retroalimentación con audios diseñados de manera pedagógica (DJ22).

Fuente: elaboración propia. 


\section{Conclusiones}

En este artículo se propuso como aporte un conjunto de directrices para el diseño, la construcción y la evaluación de juegos serios educativos, que se pretende sean incorporadas en el proceso de desarrollo de software y articuladas en las metodologías para la construcción de videojuegos, de tal modo que se propicie el desarrollo de videojuegos cada vez más pertinentes al contexto educativo deseado. Las directrices propuestas apoyan la incorporación de aspectos referentes a la usabilidad, el aprendizaje y la jugabilidad de los videojuegos serios en el contexto educativo.

En total se obtuvieron 51 directrices, organizadas de la siguiente manera: 15 de usabilidad, 13 de aprendizaje y 23 de juego; propuestas a partir de la exploración y adaptación de diferentes trabajos del estado del arte, que abordan los aspectos relacionados con la usabilidad de los videojuegos, los elementos pedagógicos presentes en los videojuegos educativos y los elementos relacionados con el juego (interactividad, motivación, logros, seguimiento, etc.).

La inspección desarrollada en el presente artículo en términos de estudio de caso permitió concluir que el videojuego serio Lighbot tiene problemas en los tres aspectos considerados en las directrices: usabilidad, aprendizaje y juego. Presenta menor número de fallas en cuanto a las directrices de aprendizaje, y un mayor número de problemas en las directrices de juego. En este último aspecto, se recomendó mejorar la retroalimentación en las acciones del jugador, la incorporación de puntajes, la inclusión de métricas como el tiempo y el nivel de cumplimiento, así como la visualización del nivel de progreso de los diferentes jugadores y la vinculación de una historia a la narrativa del juego. Como aspectos a resaltar del videojuego, se encuentran la inmersión, la secuencialidad de los retos y la promoción del aprendizaje autónomo.

Como trabajo futuro derivado de la presente investigación, se desea especificar un conjunto de directrices asociadas a la temática de accesibilidad para videojuegos serios educativos, con el fin de ampliar el número de potenciales usuarios de dichas aplicaciones. Además, se considera necesario trabajar en la generación de un conjunto de criterios de evaluación consolidados en una rúbrica que permita la conducción de evaluaciones de manera más sencilla en videojuegos serios del contexto educativo. Asimismo, se pretende extrapolar las directrices propuestas a otro tipo de categoría de juegos serios, teniendo en cuenta las generalidades y particularidades de cada contexto de aplicación.

\section{Referencias}

Abdellatif, A. J., McCollum, B. y McMullan, P. (2018). Serious games quality characteristics evaluation: The case study of optimizing robocode. SIIE 2018- 2018 International Symposium on Computers in Education, Proceedings. https://doi.org/10.1109/SIIE. 2 018.8586730

Aguilar, C., Chanchí, G., y Vidal, M. (2018). Definición de un proceso metodológico para la construcción de juegos serios para el apoyo de la enseñanza de la física cinemática. 
Publicaciones e Investigación, 12(1), 35-48. https://repository.unad.edu.co/handle/1 0596/29742

Annetta, L. A., Lamb, R. y Stone, M. (2011). Assessing serious educational games. En Serious Educational Game Assessment (pp. 75-93). SensePublishers. https://doi.org/10.1007 /978-94-6091-329-7_5

Calvo-Ferrer, J. R. (2018). Juegos, videojuegos y juegos serios: Análisis de los factores que favorecen la diversión del jugador. Miguel Hernández Communication Journal, 9, 191226. https://doi.org/10.21134/mhcj.v0i9.232

Calvo-Morata, A., Alonso-Fernández, C., Freire, M., Martínez-Ortiz, I. y Fernández-Manjón, B. (2020). Serious games to prevent and detect bullying and cyberbullying: A systematic serious games and literature review. Computers and Education, 157, 1-26. https://doi.org/10.1016/j.compedu.2020.103958

Chanchí, G. E., Gómez Álvarez, M. C. y Campo, W. Y. (2020). Propuesta de una herramienta de inspección según los atributos de usabilidad de Nielsen. Revista Ibérica de Sistemas e Tecnologias de Informação, E26, 448-460.

Da Silva Simões, P. D. y Ferreira, C. G. I. (2011). Military war games edutainment. 2011 IEEE 1st International Conference on Serious Games and Applications for Health, SeGAH 2011. https://doi.org/10.1109/SeGAH.2011.6165433

Darwesh, M. (2015). Concepts of serious game in education. International Journal of Advanced Trends in Computer Science and Engineering, 4, 15229-15232. https://doi.org/https://doi.org/10.18535/Ijecs/v4i12.25

Djaouti, D., Alvarez, J. y Jessel, J.-P. (2011). Classifying serious games: The G/P/S model. Handbook of research on improving learning and motivation through educational games: Multidisciplinary approaches. https://doi.org/10.4018/978-1-60960-495$0 . \operatorname{ch} 006$

Dörner, R., Göbel, S., Effelsberg, W. y Wiemeyer, J. (2016). Serious Games:Foundations, concepts and practice. Springer. https://doi.org/https://doi.org/10.1007/978-3-31940612-1

Enriquez, G. y Casas, S. (2013). Usabilidad en aplicaciones móviles. Informe Científico Técnico UNPA, 5(2), 25-47. https://dialnet.unirioja.es/servlet/articulo?codigo=5123524\&info= resumen\&idioma $=$ SPA

Finstad, K. (2010). The usability metric for user experience. Interacting with Computers, 22(5), 323-327. https://doi.org/10.1016/j.intcom.2010.04.004

Fitchat, L. yJordaan, D. B. (2016). Ten heuristics to evaluate the user experience of serious games. International Journal of Social Sciences and Humanity, 8(2), 209-225. 
González-Sánchez, J.-L., Montero-Simarro, F. y Gutiérrez-Vela, F.-L. (2012). Evolución del concepto de usabilidad como indicador de la calidad del software. El Profesional de la Información, 21(5), 529-536. https://doi.org/10.3145/epi.2012.sep.13

Jang, J. y Yi, M. Y. (2019). Determining and validating smart TV ux factors: A multiple-study approach. International Journal of Human Computer Studies, 130, 58-72. https://doi.org/10.1016/j.ijhcs.2019.05.001

Kramer, K.-L. (2012). Sustainability, user experience, and design. En User experience in the age of sustainability (pp. 1-30). Elsevier. https://doi.org/10.1016/b978-0-12-3877956.00001-9

López Raventós, C. (2016). El videojuego como herramienta educativa. Posibilidades y problemáticas acerca de los serious games. Revista de Innovación Educativa, 8(1), 115.

Madhavan, R. y Alagarsamy, K. (2013). Usability issues in software development lifecycle. International Journal of Advanced Research in Computer Science and Software Engineering, 3(8), 1131-1135. www.ijarcsse.com

Maheu-Cadotte, M. A., Cossette, S., Dubé, V., Fontaine, G., Deschênes, M. F., Lapierre, A. y Lavoie, P. (2020). Differentiating the design principles of virtual simulations and serious games to enhance nurses' clinical reasoning. Clinical Simulation in Nursing. https://doi.org/10.1016/j.ecns.2020.04.004

Marcano, B. (2008). Juegos serios y entrenamiento en la sociedad digital. Teoría de la Educación, 9(3),

93-107. https://gredos.usal.es/bitstream/handle/10366/56633/TE2008_V9N3_993.pdf?seque nce $=1$ \&isAllowed $=y$

Mascheroni, M., Greiner, C., Petris, R., Dapozo, G. yEstayno, M. (2012). Calidad de software e ingeniería de usabilidad. XIV Workshop de Investigadores en Ciencias de la Computación, 656-659. http://sedici.unlp.edu.ar/handle/10915/19202

Moizer, J., Lean, J., Dell'Aquila, E., Walsh, P., Keary, A. (Alfie), O’Byrne, D., Di Ferdinando, A., Miglino, O., Friedrich, R., Asperges, R. y Sica, L. S. (2019). An approach to evaluating the user experience of serious games. Computers and Education, 136, 141151. https://doi.org/10.1016/j.compedu.2019.04.006

Paliokas, I., Arapidis, C. y Mpimpitsos, M. (2011). PlayLoGo 3D: A 3D interactive video game for early programming education: Let LOGO be a game. 3rd International Conference on Games and Virtual Worlds for Serious Applications, 24-31. https://doi.org/10.1109/VS-GAMES.2011.10 
Pinelle, D., Wong, N. y Stach, T. (2008). Heuristic evaluation for games: Usability principles for video game design. Proceeding of the Twenty-Sixth Annual CHI Conference, 14531462. www.gamespot.com

Rojo, T. y Dudu, S. (2017). Los "serious games" como instrumento cultural de empoderamiento y aprendizaje socio-laboral inclusivo. Revista Fuentes, 19(2), 95-109. https://doi.org/https://dx.doi.org/10.12795/revistafuentes.2017.19.2.07

Sánchez, W. (2011). La usabilidad en ingeniería de software: Definición y características. IngNovación, 2, 7-21. https://core.ac.uk/download/pdf/47264961.pdf

Savazzi, F., Isernia, S., Jonsdottir, J., Di Tella, S., Pazzi, S. y Baglio, F. (2018). Design and implementation of a serious game on neurorehabilitation: Data on modifications of functionalities along implementation releases. Data in Brief, 20, 864-869. https://doi.org/10.1016/j.dib.2018.08.100

Tolentino, G. P., Battaglini, C., Pereira, A. C. V., De Oliveria, R. J. y De Paula, M. G. M. (2011). Usability of serious games for health. Proceedings-2011 3rd International Conference on Games and Virtual Worlds for Serious Applications, VS-Games 2011, 172-175. https://doi.org/10.1109/VS-GAMES.2011.33

Vee Senap, N. M. e Ibrahim, R. (2019). A review of heuristics evaluation component for mobile educational games. Procedia Computer Science, 161, 1028-1035. https://doi.org/10.1016/j.procs.2019.11.213

Zhu, L., Cao, Q. y Cai, Y. (2020). Development of augmented reality serious games with a vibrotactile feedback jacket. Virtual Reality \& Intelligent Hardware, 2(5), 454-470. https://doi.org/10.1016/j.vrih.2020.05.005 